(C2021 IEEE. Personal use of this material is permitted. Permission from IEEE must be obtained for all other uses, in any current or future media, including reprinting/republishing this material for advertising or promotional purposes, creating new collective works, for resale or redistribution to servers or lists, or reuse of any copyrighted component of this work in other works. This is the author's version of an article that has been published in the IEEE Communications Magazine. The final version of record is available at http://dx.doi.org/10.1109/MCOM.001.2000593 


\title{
The Role of Time in a Robotic Swarm: A Joint View on Communications, Localization and Sensing
}

\author{
Emanuel Staudinger, Siwei Zhang, Robert Pöhlmann, and Armin Dammann
}

\begin{abstract}
Autonomous robotic swarms are envisioned for a variety of sensing applications in space exploration, search-and-rescue and disaster management. An important capability of a swarm is sensing spatio-temporal processes such as radio wave propagation or seismic activities. The spatio-temporal properties of these processes dictate the required sensing position and time accuracy, as well as update rate. A dedicated wireless communication system needs to be jointly designed for swarm information exchange, self-localization and sensing. In this article, we emphasize the role of time in a robotic swarm. We introduce the system ingredients and dive into realistic clock models. Clock models and channel access schemes decisively influence the swarm self-localization and synchronization accuracy, and consequently the swarm sensing performance. Finally, we discuss practical implementation aspects, introduce our developed swarm radio system, and give an outlook on a moon-analogue exploration mission.
\end{abstract}

\section{Sensing Spatio-Temporal Processes WITH A ROBOTIC SWARM}

Autonomous robotic swarms or multi-agent systems, like illustrated in Figure 1, are envisioned for a variety of applications like space exploration, search-and-rescue and disaster management. An important asset of a swarm compared to a single robot is the capability to sense spatio-temporal processes. Examples include ocean currents, seismic activities on celestial bodies or the observation of cosmic radiation on the far side of the moon. The goal of sensing is the estimation of a set of parameters of the underlying spatio-temporal process based on jointly sensed physical quantities. Sensing accuracy is of high interest to correctly reconstruct the process, and three uncertainties must be taken into account simultaneously: sensing uncertainty due to the sensor itself, as well as uncertainty in the spatial and temporal domain, see Figure 1.

In state of the art communication systems time information plays increasingly important roles. Coarse time synchronization is required for coordinating multi-user transmission, e.g., in time-division duplex (TDD) and coordinated multipoint (COMP) based wireless communications. Channel estimation and localization demand fine time synchronization. With the development of the $5^{\text {th }}$ generation mobile networks $(5 \mathrm{G})$, especially for Ultra-Reliable LowLatency Communications (URLLC) and massive Machine Type Communications (mMTC), precise time synchronization and low latency are essential. Synchronization accuracy requirements range from tens of micro-seconds to below one micro-second, which are commonly achieved via global navigation

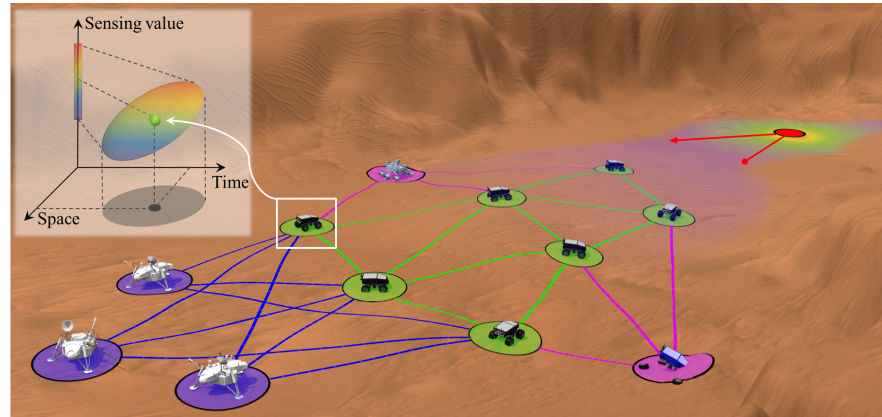

Figure 1: A robotic swarm for a future Mars exploration mission [1]: The swarm autonomously drives from the landing site to an exploration area, where a gas source is located. Agents, landers, mobility incapacitated rovers and a gas source are marked in green, blue, magenta and red, respectively. Each agent samples the spatio-temporal processes of interest with uncertainties on the sensing value, space and time, illustrated with a hyperellipsoid.

satellite system (GNSS), backbone networks, or dedicated synchronization pilots [2]. Most of the terrestrial multi-position sensing applications, such as traffic and environmental surveillance, request also time synchronization. In these applications, the timing services are provided externally by infrastructures like GNSS and cellular networks.

A swarm for exploration is often operated in an infrastructureless area, such as cave, underwater or extraterrestrial environment. The coordination of the swarm's subsystems like communication, localization, sensing and control relies on the system time generated from a mission specific swarm communication network. As a consequence, the time information in a swarm is deeply coupled with the aforementioned subsystems, which leads to different timing criteria in comparison with state of the art systems.

In this article, we shed light on the role of time in a swarm in infrastructureless environments, which decisively determines the swarm exploration performance. The required time synchronization and selflocalization accuracy is determined by the missionspecific sensing application. Figure 2 gives insight on requirements for a variety of sensing applications. We identify radio-based sensing as the most demanding one, both in terms of sensing time- and position accuracy. Radio-based sensing applications are for example low frequency arrays (LOFARs), and return-to-base navigation for a robotic swarm. A LOFAR is a distributed coherent antenna array 
for radio astronomy, and in return-to-base navigation the robotic swarm shall navigate back to the lander through observing a low-frequency radio beacon. Decimeter-level sensing position accuracy and nanoseconds sensing time accuracy are required for radio-based sensing applications, which are not achievable with state of the art communication systems in GNSS-denied environments. We introduce building blocks for a swarm system, incrementally develop the role of time, and show fundamental dependencies among those building blocks. We also provide insights and examples on the design of a mission-specific wireless communication system for swarm exploration, and introduce our real-world swarm system.

\section{SWARM System BUILDING BlOCKS}

As discussed previously, designing such a swarm system, particularly the mission-specific communication system, requires a joint look at all the building blocks, which we describe next.

\section{A. Communication}

Communication among agents is required to enable collaborative behavior. Self-organized meshed adhoc communication networks are preferred to avoid single-point of failures. Distributed algorithms for self-localization and sensing are mission-specific and may require a low latency depending on swarm mobility and the sensed spatio-temporal process. The communication system's physical layer (PHY) and media access control layer (MAC) with its channel access scheme, and protocols must meet those requirements. The design of such PHY and MAC for broadcast-based meshed ad-hoc communication is challenging.

\section{B. Self-Localization}

In exploration it is important to know where sensors carried by robots are located within the exploration area to map measured physical quantities. Knowledge of position in three dimensions is sufficient to map scalar fields like temperature or pressure and sensing vector fields like electromagnetic fields or wind/water currents additionally requires knowledge about the orientation. Control algorithms for a robotic swarm need to know position and preferably orientation of agents as well. Requirements on selflocalization accuracy are determined by the sensing application and control building blocks. Wireless communication systems are utilized twofold in swarm self-localization. Firstly, the wireless components can be considered as localization sensors. The geometrical information among agents, e.g., distance and angle, can be extracted from the signal propagation properties like signal delay and carrier phase. Secondly, agents use the communication system to exchange their position and time estimates, as well as the corresponding uncertainties, which is

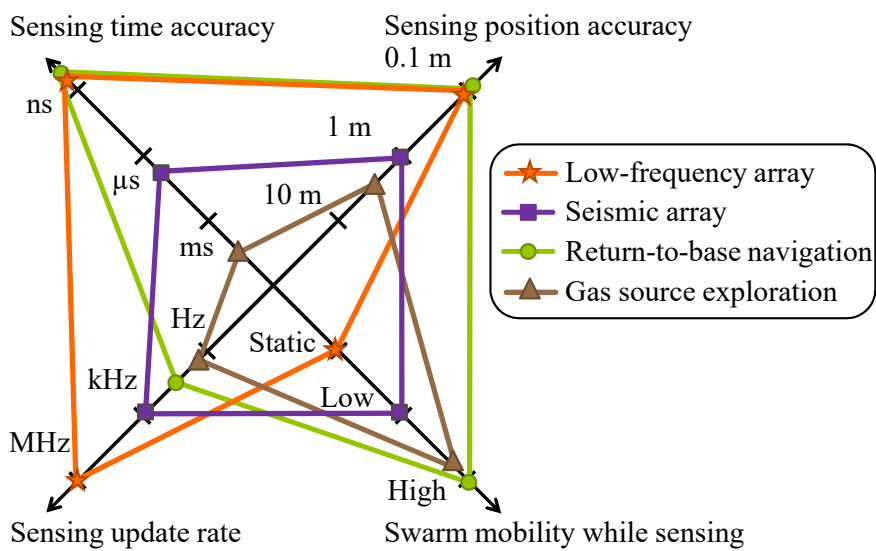

Figure 2: Typical requirements for selected robotic swarm sensing applications.

essential particularly for decentralized localization algorithms [3], [4].

\section{Sensing}

The fundamental task of exploration is the reconstruction of a spatio-temporal process through parameter estimation based on sensed physical quantities. Spatio-temporal processes show dependencies in space and time which are described by appropriate models. Depending on the model, physical quantities have to be taken sufficiently dense with respect to space and time. Expected process change rates, described by partial first order derivatives, proportionally set the accuracy requirements for self-localization and timing building blocks, and determine communication latency.

\section{Control}

Control algorithms steering the individual agents aim to optimize the exploration progress by, for instance, maximizing the information gain in each control step while taking all constraints and requirements into account. Besides localization, sensing and timing accuracy requirements, control algorithms also have to take into account safety aspects like collision avoidance when operating in challenging environments.

\section{E. Timing}

All of the building blocks addressed before need knowledge about time among all agents with certain accuracy. Precise localization using radio signal propagation delay based methods requires accurate timing in the order of nanoseconds or even below for signal time of arrival measurements. Timing requirements for communications are less strict and for the control part it depends on the dynamics of the swarm system itself, see Figure 2. Requirements on timing accuracy from sensing applications depend on the dynamics of the spatio-temporal process to be observed. These requirements may range from sub-nanoseconds for the observation of elec- 
tromagnetic waves up to the order of seconds when observing slowly varying temperature fields, see Figure 2. However, localization accuracy requirements indirectly determine timing requirements for propagation delay based localization.

\section{The Role of ClOCKS}

Clocks play a fundamental role for time synchronization, localization, and as we will see, communication system design. The frequency of an underlying oscillator of a clock is a function of the type of oscillator and its physical properties [5]. Clocks are characterized through relative values: time differences and fractional frequency difference between a device under characterization and an ideal one. Two key metrics must be taken into account, which we discuss next: Frequency accuracy and the stability of frequency.

Frequency accuracy is denoted as the fractional frequency relative to the nominal frequency, and is a unitless quantity [5]. The value of this quantity depends on the type of oscillator technology. A low-cost temperature controlled crystal oscillator (TCXO) has a typical frequency accuracy of $\pm 10 \mathrm{ppm}$ resulting in a frequency offset of $\pm 100 \mathrm{~Hz}$ for a $10 \mathrm{MHz}$ oscillator.

The stability of frequency depends on noise sources within the oscillator. A common method to capture this stability, and consequently the ability to predict the clock, is the overlapping Allan deviation (OADEV). The OADEV is denoted as square-root of the two-sample variance of time differences for a commonly called averaging interval $\tau$. Figure 3 shows exemplary OADEVs for different oscillators in order of increasing stability: TCXO, microelectromechanical systems (MEMS), oven-controlled crystal oscillator (OCXO), and Rubidium. The TCXO has been characterized with our swarm radio system, and the OCXO and Rubidium clock have been characterized in [6]. OADEV curves show three distinct regions over $\tau$ which are of interest for us: A decreasing OADEV for small averaging intervals to a minimum or flat region, and an increasing OADEV for larger $\tau$. Short-term and long-term stability are affected by different noise sources within the oscillator and its physical properties [5]. Increased stability comes with increased cost per device and larger power consumption. We focus on two important findings only: Firstly, the minimum value at a specific averaging interval. Secondly, the averaging interval at which the OADEV turns into a $+1 / 2$ or +1 slope.

The averaging interval $\tau$ can equivalently be seen as an observation interval, which results in two practical implications. 1) The minimum OADEV is a lower limit on the clock estimation uncertainty. 2) The averaging interval $\tau$ at the transition to $\mathrm{a}+1 / 2$ or +1 slope is an upper limit on the observation interval to adequately estimate and track the clock.

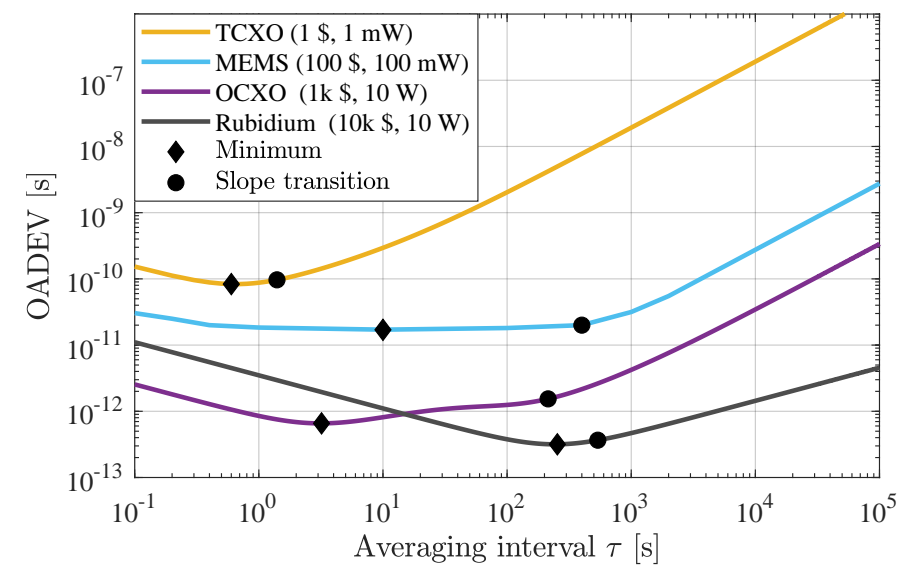

Figure 3: Exemplary OADEVs of selected clocks: A low OADEV over $\tau$ is desired. Depicted costs and power demands are shown in the order of magnitude.

An observation interval beyond that results in a degraded clock estimation accuracy. The update rate of clock observations determined by the channel access scheme is important for 1) and 2). For example, using the TCXO in Figure 3 limits the minimum update rate for the wireless communication system to $1 / 1.5 \mathrm{~Hz}$ to still be able to track the clock adequately. By using MEMS we can decrease the update rate to $1 / 400 \mathrm{~Hz}$, and we therefore only need to access the mobile radio channel once in $400 \mathrm{~s}$ resulting in less energy required.

From this section we can conclude that the stability of the used clock is key for the design of a wireless communication system, and we refer the interested reader to [6] on the state-space representation and simulation of clocks.

\section{TIME INFORMATION IN SWARM SELF-LOCALIZATION}

Localization in network is a well addressed topic in literature from theoretical limits [7] to algorithm design [3], [8]. There is little research on a joint look at localization and clock. Simultaneous localization and synchronization is discussed in [9], whereas localization accuracy and latency tradeoff is discussed in [10], both with simplified clock models. Besides, most of these research assume an established communication system. In this section, we focus on the impact of realistic clock models on localization, which decisively determines the PHY and MAC design of the underlying mission-specific wireless communication system.

Extracting distance information from signal intensity like received signal strength (RSS) or signal delay, is referred to as ranging [11]. RSS based ranging is independent from any clock information. It simplifies the ranging implementation but cannot inherently provide the essential time information to the swarm. More importantly, RSS based ranging is sensitive to the applied propagation model, and thus, often associated with insufficient ranging accuracy 
for swarm applications. In contrast, signal delay based ranging offers both accurate distance and time information. Depending on the synchronization condition of the network, a variety of delay based methods can be applied. Time of arrival (ToA) measurement assumes a fully synchronized network. The impact of clock on ranging is neglected in ToA, which simplifies the theoretical investigation [7]. In a partially synchronized network, time difference of arrival (TDoA) measurements can be obtained, which are used in hyperbolic localization of an asynchronized agent. TDoA is widely applied in infrastructure based localization like GNSS and cellular localization. For an asynchronized network, round trip time (RTT) measurements assume a simplified clock model and combine the signals on both directions of a link to eliminate the clock offsets. Traditionally, unicast communication schemes are employed for RTT like in the IEEE $802.11 \mathrm{mc}$ standard [12]. Hence, the reply signal is directly transmitted upon reception of the initiation signal. With a short reply time, e.g., in the order of microseconds, the impact of clock frequency offset and stability of clocks are insignificant. This scheme performs well for small networks and for anchorbased localization. However, for an asynchronized large meshed network like a swarm, the unicast scheme results in low update rates in localization. We extend the idea of RTT to a broadcast communication scheme, where, for example, agents transmit signals sequentially with a self-organized timedivision multiple access (TDMA) frame structure. In this scheme, we benefit from higher update rates at the cost of a longer reply time, where the clock impacts cannot be neglected. Thus, the clock parameters are continuously tracked with a realistic clock model, alongside the distance. As a comparison, with unicast, $N(N-1)$ transmissions (quadratic to the number of agents $N$ ) are required to compose a fully connected network, whereas with broadcast only $N$ transmissions (linear to the number of agents $N)$ are required.

We have developed a distributed particle filter (DPF) in [4], which is proved in experiments particularly suitable for swarm self-localization. In [1] we have demonstrated algorithm design for simultaneous swarm self-localization, synchronization, sensing and information seeking control. To gain better insights in the relation between localization and clock, we investigate a simplified, yet instructive example in this article. We model the range between agents as a second order process with a white noise acceleration model with standard deviation of $1 \mathrm{~m} / \mathrm{s}^{2}$. Channel access for ranging measurements with $10 \mathrm{~dB}$ signal to noise ratio (SNR) is realized via TDMA with a slot length of $1 \mathrm{~ms}$. Localization performance is, in general, jointly determined by the ranging quality and network geometry. In order to focus on the clock and channel access aspects, we

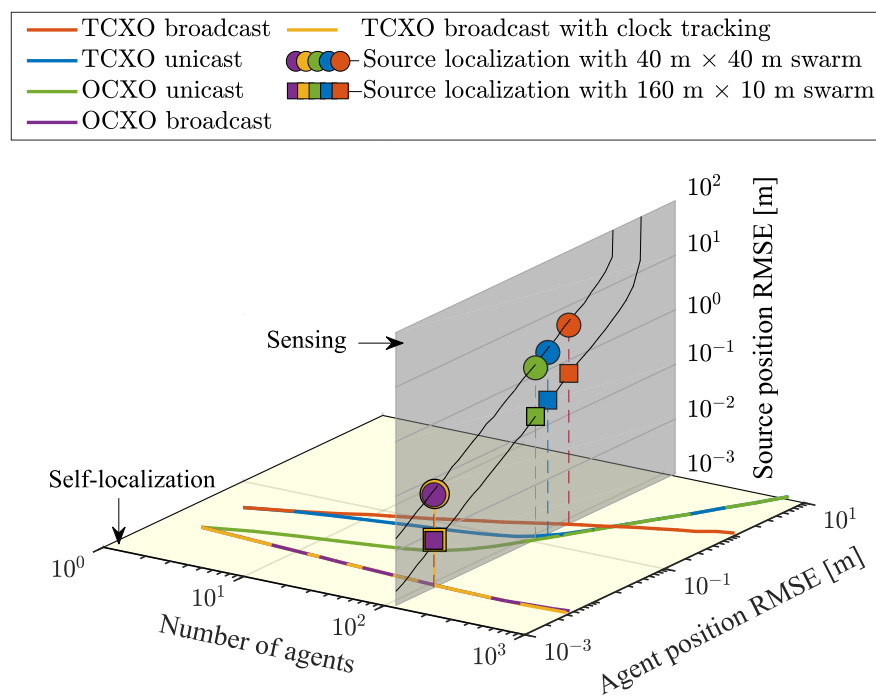

Figure 4: Swarm self-localization and sensing are closely linked: The $x$ - $y$-plane contains the expected agent position root mean square errors (RMSEs) for a fully connected network of variable size given different clock types and channel access schemes, whereas the source position RMSEs are plotted for two different swarm formations on the $y$-z-plane.

exploit a theorem from [7]: For a fully connected network of $N$ agents, the position RMSE is expected to be of the same order of magnitude as the ranging RMSE scaled by $\sqrt{2 / N}$. The result is shown in Figure 4 , where we focus on the $x$ - $y$-plane, i.e. number of agents vs. expected agent position RMSE. The $y$ $z$-plane refers to sensing and is discussed in the next section. No clock tracking is applied for the unicast scheme as well as for the broadcast scheme, and we want to compare it with the broadcast scheme with clock tracking. Let us have a look at the TCXO first. For two agents, unicast and broadcast without clock tracking are identical. When the number of agents is increased, first unicast has an advantage due to shorter RTTs, and thus less error from the fractional clock frequency offset. For a large number of agents, unicast becomes worse due to long update intervals scaling quadratically with the number of agents and the assumed white noise acceleration model for swarm mobility. Broadcast with appropriate clock tracking always performs better and shows position RMSE improvements in the order of magnitudes, since the fractional clock frequency is estimated and compensated. The stability of OCXOs is roughly two orders of magnitude better than for TCXOs, see Figure 3. OCXO unicast starts at a lower RMSE for two agents than TCXO unicast, but for a large number of agents the curves unite. This performance gap between the two clock types depends on the slot length; for shorter slots TCXO unicast performance approaches OCXO unicast. For OCXO broadcast without clock tracking we obtain a similar position accuracy compared to TCXO broadcast with clock tracking. 
For RTT based swarm self-localization we can conclude that unicast schemes are not well suited for a larger number of agents, and broadcast schemes are preferable for large robotic swarms if the real clock is appropriately taken into account. The TDMA slot length, number of agents, and the used clock need to be considered jointly. One can select more stable clocks which come with increased cost in terms of pricing, weight, and power demands.

As an outlook, other impacts of time on localization performance are worth further investigation. In decentralized localization algorithms, the amount of exchanged data and the number of algorithm iterations till convergence result in additional latency [3], [4]. In [10], passive agents localization with TDoA besides of RTT is also discussed, which potentially increases the localization update rate.

\section{TIME INFORMATION IN SWARM SENSING}

The swarm sensing strategies should be designed according to the dynamics of the spatio-temporal processes, which are continuous functions of position and time. To get an intuition of the dynamics of different processes, let us take an example of two agents which are located $10 \mathrm{~m}$ apart. A radio or light signal can propagate across the two agents in $33.3 \mathrm{~ns}$. For acoustic or seismic waves, it will take $3 \mathrm{~ms} \sim 30 \mathrm{~ms}$. Considering fluid dynamics, e.g., airflow or ocean currents, the propagation time across the two agents will be $0.3 \mathrm{~s} \sim 3 \mathrm{~s}$. The performance of the spatio-temporal process reconstruction is jointly determined by 1) the spatial and temporal sensing density, 2) the uncertainties in sensing value, sensing time and sensor position, and 3 ) the position and time of sensing.

1) Observations have to be taken sufficiently dense with respect to space and time according to the Nyquist theorem to reconstruct these continuous functions. Hence, agents must be placed densely enough to capture the spatial variation of these functions. Similarly, the sampling rate at each agent has to be sufficiently high to capture the temporal dynamics of these functions. Particularly for a process describing a propagation phenomenon like radio wave propagation, spatio-temporal correlation can be exploited. In this case, the process can be reconstructed with coherent spatial sampling only.

2) The exact position and time at which an agent observes physical quantities are unknown and thus have to be estimated. As illustrated in Figure 1 , the performance of spatio-temporal process reconstruction is jointly determined not only by the uncertainty of the sensed value, but also by the agent's uncertainties in position and time. The necessary accuracy of swarm self-localization and time synchronization are determined by the dynamics of the spatio-temporal process, similarly as for the sampling density.

3) The actual sensing position and time can be optimized to achieve high sensing accuracy, which leads to the topic of swarm control. Information seeking control can be considered, where a swarm's formation is optimized to improve swarm self- and source localization performance [1].

A practical challenging example is low frequency radio frequency (RF) source localization, see Figure 2. Let us assume a swarm shall localize an RF source, which is located $100 \mathrm{~m}$ away from the swarm center. The RF source emits a periodic carrier signal with a wavelength of $\lambda=16 \mathrm{~m}$. We compare two swarm formations, a $40 \mathrm{~m} \times 40 \mathrm{~m}$ square formation with 121 agents and a $160 \mathrm{~m} \times 10 \mathrm{~m}$ rectangular formation with 124 agents, where the longer edge is perpendicular to the source direction. Agents are spatially separated from each other by a quarterwavelength $\lambda / 4=4 \mathrm{~m}$. With a spherical-wave model, the source angle of arrival (AoA) and distance, and thus, position can be estimated by observing the first and second spatial derivative of the signal carrier phase [13]. To highlight the link between self-localization and sensing, we have included the sensing performance in Figure 4. Now, we focus on the $z$-y-plane of Figure 4, which shows the source position estimation performance of a maximum likelihood (ML) estimator over swarm selflocalization and time synchronization accuracy. The self-localization accuracy for a certain clock and ranging scheme corresponds to a specific sensing accuracy for the source position. With a low-cost TCXO, only the broadcast ranging scheme with clock tracking can achieve centimeter level source position RMSE. A similar source localization accuracy can be achieved with a more stable OCXO by broadcast ranging schemes without clock tracking. The rectangular swarm formation results in a more precise source localization due to a larger effective aperture perpendicular to the source direction. This highlights the potential improvement by optimizing the swarm formation taking the control aspect into account [1].

It can be seen that the time information decisively affects the swarm sensing, both directly through the update rate and time synchronization, and indirectly through swarm self-localization. By employing broadcast schemes with appropriate clock tracking, low-cost clocks like TCXOs can be sufficient for precise swarm sensing applications.

As an outlook, a joint view on swarm localization, synchronization and control is worth further investigation. Distributed sensing algorithms, like consensus or diffusion based ones, are particularly of interest for robotic swarm systems [14]. These algorithms require additional iterations with data exchange among agents to reach convergence. 


\section{PAVING the Way to a Real-World SWARM MISSION}

\section{A. Innovation and Development}

We have been developing a series of swarm prototypes with our mission-specific wireless communication system concept and a multi-agent eco-system. The system is optimized for swarm communication, localization, timing, sensing and control, particularly suitable for future planetary surface exploration missions. Our design is driven by the following aspects: The expected radio channel on martian or lunar surface, mobility of agents, spatio-temporal characteristics of the sensed process, properties of available light-weight and low-cost software-defined radios (SDRs), robustness against agent failures, and simplified interfacing in the swarm eco-system.

For the communication system, on PHY we use orthogonal frequency-division multiplexing (OFDM) with $25 \mathrm{MHz}$ bandwidth, 1024 subcarriers and a cyclic-prefix of $2 \mu \mathrm{s}$. On MAC we implement a collision-free self-synchronized TDMA broadcast scheme, which avoids single-point of failure and results in a controllable update rate. The update rate is driven by the distributed algorithms for localization and sensing, the mobility of the swarm in conjunction with spatio-temporal correlation of process parameters, and the used clocks. Distributed algorithms often require iterations among agents to converge, and hence, the radio channel must be accessed frequently. We design for a network update rate of $100 \mathrm{~Hz}$ for 20 agents resulting in slot lengths of $500 \mu \mathrm{s}$ and $11 \mathrm{OFDM}$ symbols per agent. An agent-specific preamble based on Zadoff-Chu codes is used for frame-synchronization, channel estimation, agent-identification, and precise estimation of a frame's arrival time for ranging and clock tracking. This preamble is designed for differential correlation resulting in low processing effort to maintain the TDMA frame structure. The remaining 10 OFDM symbols can be flexibly exploited for inter-agent communications. Each agent tracks the dynamics of its clock and the clocks of its neighboring agents with Kalman filters (KFs) to compensate fractional frequency offsets. The KF employs the transition model parameters of the clocks [6], like the TCXOs embedded in SDRs, obtained from RF calibration. One SDR is connected to an external Rubidium clock as a stable frequency reference. As an outcome of the laboratory calibration, the OADEV of the TCXO can be found in Figure 3. We make use of SDRs comprising various types of universal software radio peripherals (USRPs) and a custom PHY and MAC realized in the open-source framework Gnuradio. Different types of USRPs have different clocks, for example the light-weight B200mini uses an embedded TCXO, and a multichannel N310 uses either the embedded OCXO or an external clock. Our design enables the usage of heterogeneous hardware and takes properties of

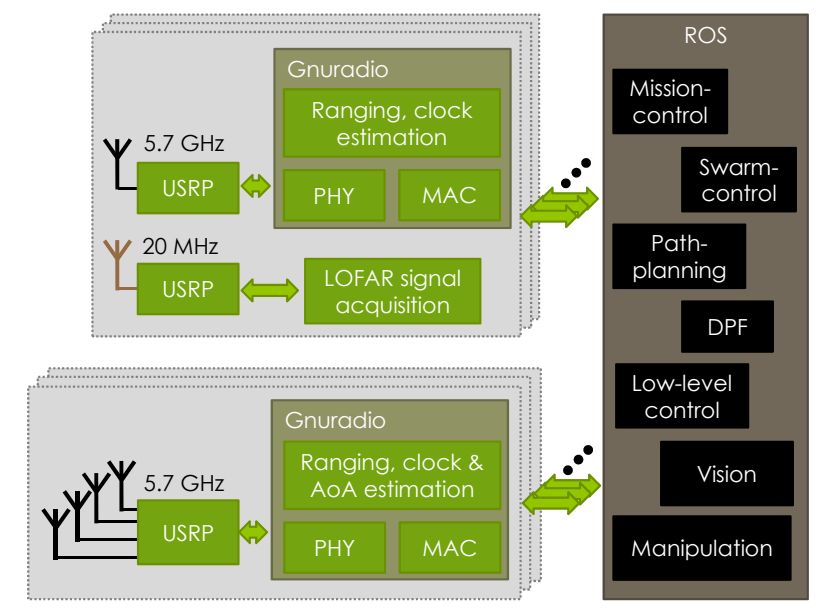

Figure 5: Architecture of our swarm eco-system based on SDRs and the ROS.

individual clocks into account.

A challenge besides the design of PHY and MAC is the interfacing into the other swarm subsystems. We utilize the robot operating system (ROS), which is commonly used in robotics, to tackle this challenge. A DPF is implemented on each agent for realtime self-localization, exploiting the ranging information or direct radio waveform from the SDR [4], and potentially other sensor outputs in ROS. The DPF provides the estimated position and time with their uncertainties to the other subsystems in ROS, such as swarm sensing and control. The overall architecture of our swarm eco-system is shown in Figure 5.

\section{B. Experiment Highlights}

Figure 6 shows the evolution of hardware development at German Aerospace Center (DLR). Our first version in 2015 was based on a dedicated fieldprogrammable gate array (FPGA) design, and we focused on unicast based RTT with an amplify-andforward scheme, which leads to short processing delays. For a six-agent swarm with low dynamics, decimeter-level localization accuracy is achieved with our DPF by directly exploiting the radio waveform [4]. Since 2017 we realize the concept from Figure 5 and demonstrated real-time submeter-level localization accuracy with eight agents for a highly dynamic gas source exploration. In 2018 we demonstrated swarm self-localization at the International Astronautical Congress (IAC) [15]. In June 2021 a distributed LOFAR demonstration with our swarm eco-system is planned within a moon-analogue exploration mission taking place on Mt. Etna in Sicily, Italy [15]. As a preparation, the mission-specific communication system has been preliminarily tested on Mt. Etna during a scouting mission in 2019.

\section{CONCLUSION}

Time is key for a swarm mission, and the inclusion of realistic clock modeling is of high importance. 


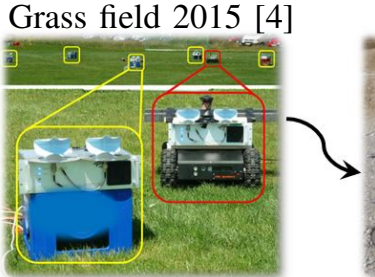

First tests on Mt. Etna 2019

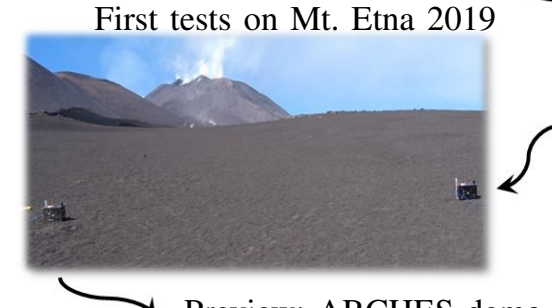

Preview: ARCHES demo on Mt. Etna 2021 [15]

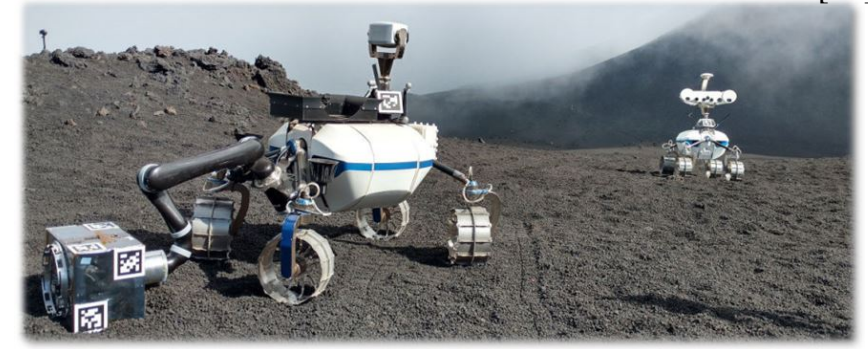

Figure 6: Generations of our swarm radio system developed, tested in field and demonstrated at the IAC. The latest generation will be part of a moon-analogue mission on Mt. Etna, Italy, in 2021.

The design of a mission-specific wireless communication system is jointly driven by the clock model, swarm dynamics, localization requirements and the properties of the spatio-temporal process.

Particularly the MAC design and the stability of the oscillator, from which time is derived from, are strongly coupled. The update rate of clock observations determines time synchronization accuracy, which has a decisive impact on self-localization, and consequently, on the sensing accuracy in both position and time domains.

Regarding a real robotic swarm system, joining effort in technology demonstration across research domains of wireless communication and robotics within a unified eco-system is promising and should be fostered.

\section{REFERENCES}

[1] S. Zhang, R. Pöhlmann, T. Wiedemann, A. Dammann, H. Wymmeersch, and P. A. Hoeher, "Self-aware swarm navigation in autonomous exploration missions," Proc. IEEE, vol. 108, no. 7, pp. 1168-1195, 2020.

[2] V. Jungnickel, K. Manolakis, W. Zirwas, B. Panzner, V. Braun, M. Lossow, M. Sternad, R. Apelfröjd, and T. Svensson, "The role of small cells, coordinated multipoint, and massive mimo in 5g," IEEE Commun. Mag., vol. 52, no. 5, pp. 44-51, 2014

[3] H. Wymeersch, J. Lien, and M. Win, "Cooperative localization in wireless networks," Proc. IEEE, vol. 97, no. 2, pp. 427-450, Feb. 2009.

[4] S. Zhang, E. Staudinger, T. Jost, W. Wang, C. Gentner, A. Dammann, H. Wymeersch, and P. A. Hoeher, "Distributed direct localization suitable for dense networks," IEEE Trans. Aerosp. Electron. Syst., vol. 56, no. 2, pp. 1209-1227, 2020.
[5] J. Levine, "Introduction to time and frequency metrology," Review of Scientific Instruments, vol. 70, no. 6, pp. 2567-2596, 1999.

[6] C. Trainotti, T. Schmidt, and J. Furthner, "Comparison of clock models in view of clock composition, clock steering and measurement fitting," in Proc. of the 50th Annu. Precise Time and Time Interval Syst. and Appl. Meeting, Reston, USA, January 2019, pp. 265-283.

[7] Y. Shen, H. Wymeersch, and M. Win, "Fundamental limits of wideband localization, part II: Cooperative networks," IEEE Trans. Inf. Theory, vol. 56, no. 10, pp. 4981-5000, Oct. 2010.

[8] R. M. Buehrer, H. Wymeersch, and R. M. Vaghefi, "Collaborative sensor network localization: Algorithms and practical issues," Proc. IEEE, vol. 106, no. 6, pp. 1089-1114, Jun. 2018.

[9] B. Etzlinger, F. Meyer, F. Hlawatsch, A. Springer, and $\mathrm{H}$. Wymeersch, "Cooperative simultaneous localization and synchronization in mobile agent networks," IEEE Trans. Signal Process., vol. 65, no. 14, pp. 3587-3602, 2017.

[10] G. E. Garcia, L. S. Muppirisetty, E. M. Schiller, and $\mathrm{H}$. Wymeersch, "On the trade-off between accuracy and delay in cooperative UWB localization: Performance bounds and scaling laws," IEEE Trans. Wireless Commun., vol. 13, no. 8, pp. 45744585,2014

[11] D. Dardari, A. Conti, U. Ferner, A. Giorgetti, and M. Z. Win, "Ranging with ultrawide bandwidth signals in multipath environments," Proc. IEEE, vol. 97, no. 2, pp. 404-426, Feb. 2009.

[12] G. Guo, R. Chen, F. Ye, X. Peng, Z. Liu, and Y. Pan, "Indoor smartphone localization: A hybrid WiFi RTT-RSS ranging approach," IEEE Access, vol. 7, pp. 176767-176781, 2019.

[13] S. Zhang, T. Jost, R. Pöhlmann, A. Dammann, D. Shutin, and P. A. Hoeher, "Spherical wave positioning based on curvature of arrival by an antenna array," IEEE Wireless Commun. Lett., vol. 8, no. 2, pp. 504-507, Apr. 2019.

[14] A. H. Sayed, "Adaptive networks," Proc. IEEE, vol. 102, no. 4, pp. 460-497, 2014

[15] M. J. Schuster et al., "The ARCHES space-analogue demonstration mission: Towards heterogeneous teams of autonomous robots for collaborative scientific sampling in planetary exploration," IEEE Robot. Autom. Lett., vol. 5, no. 4, pp. 5315-5322, 2020 .

Emanuel Staudinger received a M.Sc. in Embedded Systems Design from the University of Applied Sciences of Hagenberg, Austria, in 2010, when he joined the Institute of Communications and Navigation of the German Aerospace Center (DLR), Munich, Germany as Research Staff Member. He received a Ph.D. with distinction from the Institute of Electrodynamics and Microelectronics of the University of Bremen, Germany, in 2015. His current research interests include system design for cooperative positioning, experimental platform design based on SDRs, and experimental validation for swarm navigation.

Siwei Zhang received the B.Sc. in electrical engineering from the Zhejiang University, China, in 2009, the M.Sc. in communication engineering from the Technical University of Munich, Germany, in 2011, and the Dr.-Ing. (Ph.D.) in electrical engineering from the University of Kiel, Germany, in 2020. In 2012, he joined the Institute of Communications and Navigation, German Aerospace Center (DLR), Germany, as a Research Staff Member. His research interests include radio navigation, cooperative positioning and swarm navigation.

Robert Pöhlmann received B.Sc. and M.Sc. in electrical engineering and information technology from the Technical University of Munich (TUM), Germany, in 2014 and 2016, respectively. In 2013, he joined the Institute of Communications and Navigation, German Aerospace Center (DLR), Germany, as a Student Trainee, where he became a Research Staff Member in 2016. His current research interests are in the area of statistical signal processing for multi-antenna systems and cooperative localization.

Armin Dammann received the Dipl.Ing. (M.Sc.) and Dr.Ing. (Ph.D.) in electrical engineering from the University of Ulm, Germany, in 1997 and 2005, respectively. In 1997, he joined the Institute of Communications and Navigation, German Aerospace Center (DLR), Germany, as a Research Staff Member, where has been the head of the Mobile Radio Transmission Research Group since 2005. His research interests include signal design and signal processing for terrestrial wireless communications and navigation systems. 